K.A. van der Hucht, G. Koenigsberger \& P.R.J. Eenens, eds.

\title{
Colliding stellar winds: recent advances in modelling
}

\author{
Ian R. Stevens and Julian M. Pittard \\ The University of Birmingham, Edgbaston, Birmingham B15 2TT, UK
}

\begin{abstract}
We report on recent advances in the modelling of colliding stellar winds in WR binaries. Here, we concentrate on the modelling of X-ray observations using numerical gas-dynamics, in some cases including radiation hydrodynamics, and look in detail at a few systems, such as $\gamma^{2}$ Velorum, $\eta$ Carinae and HD 193793. We also report briefly on the modelling of infrared line-profile variability in a number of close WR+O-star systems, which can reveal further information on the dynamics of the interaction region. We discuss recent 3Dsimulations and describe the exciting prospects that $A X A F$ and $X M M$ open up.
\end{abstract}

\section{Introduction}

For winds with terminal velocities typical of OB or Wolf-Rayet (WR) stars, passing through the strong shocks expected in colliding wind systems, the postshock temperatures will be $\sim 10^{7-8} \mathrm{~K}$. Consequently, the post-shock material will emit at X-ray energies, and so X-ray observations will be the prime method of observing the post-shock flow in colliding wind systems. In this paper we report on recent modelling developments of the colliding wind phenomena in WR binary systems and related X-ray observations. We concentrate on a few key colliding wind systems. In addition, we shall briefly report on near IR lineprofile observations which also sample the post-shock region.

\section{X-ray observations and modelling of colliding wind systems}

\section{1. $\gamma^{2}$ Velorum}

$\gamma^{2}$ Vel holds a special place in the study of WR stars, being both the closest and brightest WR star. It is a binary system with $P_{\text {orb }}=78.5$ days, and an eccentricity of $e \simeq 0.3$. $\gamma^{2}$ Vel has been the subject of detailed study at X-ray energies, yielding much insight into colliding winds. X-ray observations with ROSAT (Willis et al. 1995) showed a lightcurve that was flat for most of the orbit, but which exhibited a strong rise in emission around periastron passage. Further observations revealed that this rise in emission repeated from orbit to orbit, and so was not some transient flare phenomenon.

The model adopted by Willis et al. (1995) to explain this rise in flux around periastron invoked colliding winds. For the majority of the orbit, X-ray emission from the colliding stellar winds was almost completely absorbed by the dense WR wind. The nuclear processed composition of WR winds means that WC wind material is a very efficient absorber of soft X-rays (primarily due to enhanced C 

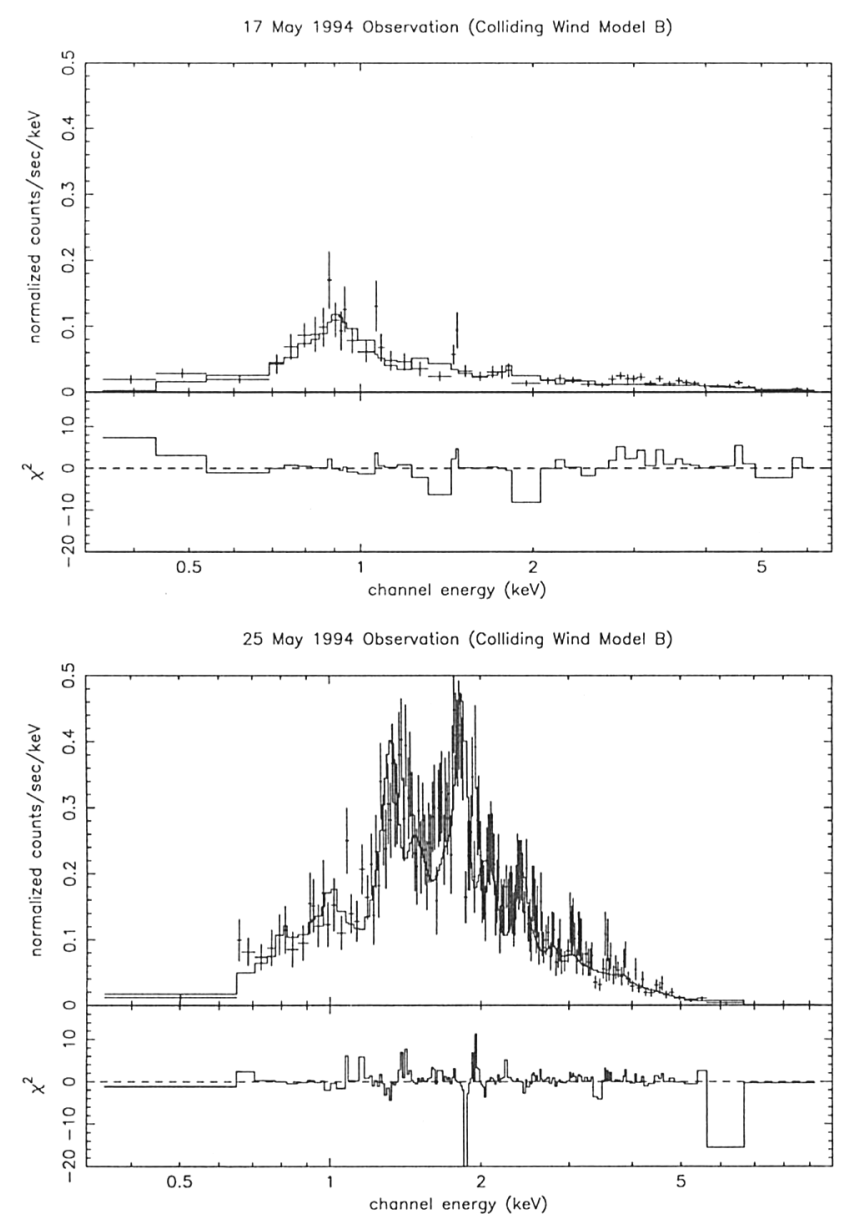

Figure 1. ASCA observations of $\gamma^{2}$ Vel at phase $\phi=0.4$ (top) and 0.5 (bottom).

and $\mathrm{O}$ abundances). Close to periastron the line-of-sight switches from the WR wind to that of the O-type star. The absorbing column drops rapidly, allowing much more X-ray flux from the wind interaction to escape.

The ASCA satellite has some advantages over ROSAT, in that it has much better spectral resolution (it also has much worse spatial resolution but for point sources this is less of an issue). To further investigate the colliding wind emission from $\gamma^{2}$ Vel we obtained two ASCA observations at phases $\phi=0.4$ and $0.5^{1}$ and results have been reported in Stevens et al. (1996). The data from these observations are shown in Figure 1 , and show very substantial variability between the observations. At $\phi=0.5$, in particular, we see strong emission, with evidence of line emission in the spectrum.

\footnotetext{
${ }^{1}$ Note, that there are different definitions of orbital phases in $\gamma^{2}$ Vel. In our definition phase $\phi=0.5$ is when the O-type star is in front of the WR star.
} 
For $\gamma^{2}$ Vel it seems clear that during the rise in X-ray emission around periastron the observed $\mathrm{X}$-ray emission is primarily due to colliding winds. The luminosity and spectral shape of the X-ray emission will therefore depend on the wind characteristics (such as mass-loss rate and velocity) of both stars. For instance, a higher wind velocity will, in general, result in hotter spectra, and a higher mass-loss rate generally in a higher luminosity. Consequently, we should be able to investigate the stellar wind characteristics from the X-ray emission.

In Stevens et al. (1996) we made the first attempt at using hydrodynamical models of colliding winds to model and fit the data. The procedure used was as follows. First we calculated a series of theoretical colliding models for a range of parameters. We assumed the orbit was well known and calculated models with different mass-loss rates and terminal velocities for both stars. The hydrodynamic simulations were performed using $2-\mathrm{D}$ terminal velocity simulations (i.e. no radiative driving). For a particular orbital phase we calculate a grid of colliding wind hydrodynamic models for different mass-loss rates/wind velocities, and then from each model we calculate synthetic X-ray spectra (which will be a complex multi-temperature, multi-column spectrum). Then we fit the $A S C A$ spectra with the synthetic spectra to determine a best-fit. Perhaps not surprisingly, the results were largely inconclusive. However, one strong constraint was made, namely that the mass-loss rate of the WR component was constrained to be $\dot{M}_{\mathrm{WR}}=2-3 \times 10^{-5} \mathrm{M}_{\odot} \mathrm{yr}^{-1}$. This is an interesting result, somewhat lower than previous determinations, but in line with recent radio determinations (Chapman et al. 1999) However, we should note that this X-ray determination of mass-loss rates should be regarded with a certain amount of caution, and much more work is needed to make it a reliable technique.

\section{2. $\eta$ Carinae}

$\eta$ Carinae is perhaps the most luminous and massive star in the galaxy. Recent optical observations suggest that the system is a binary (Damineli 1996), with a period of 5.5 years and a large eccentricity. Long-term monitoring of the Xray behaviour of $\eta$ Car with ROSAT, ASCA and RXTE reveal a remarkable lightcurve (Corcoran et al. 1995, 1998), showing a steep rise in emission up to periastron passage and then a precipitous drop immediately afterwards. While evidence of the nature of the companion is still lacking there is a high probability that it is also a massive star with a strong stellar wind, making $\eta$ Car a colliding wind system.

To investigate the possible impact of colliding winds we have calculated a synthetic X-ray light curve for $\eta$ Car (see Pittard et al. 1998). We assume an LBV primary with a very massive, but relatively slow wind, and an O-star companion with a much faster but less massive wind.

We find that a straightforward colliding wind model can explain many of the observed features of the X-ray lightcurve. The long rise up in flux towards periastron is a simple consequence of X-ray emission from colliding winds as the stars approach each other. The sharp dip near periastron is a consequence of the system orientation. Around periastron the line of sight from the X-ray emitting zone will be hidden behind the dense LBV wind and largely obscured, resulting in a sharp fall. Some features remain to be explained, namely the asymmetric 
recovery in the X-ray lightcurve. However, the inclusion of 3D effects in models of $\eta$ Car may well be able to explain this.

\subsection{HD 193793 (WR 140)}

HD 193793 is often cited as another prime example of a colliding wind system. It is a wide, eccentric binary (WC7+O $4-5, P_{\text {orb }}=7.9 \mathrm{yr}, e=0.85$, Williams et al. 1990). The source is famous for showing periodic and enigmatic episodes of dust formation associated with periastron passage. It is also a strong and variable X-ray source, and has been monitored at X-ray energies, with ROSAT and $A S C A$, for several years. The latest results are discussed by Corcoran et al. (these Proceedings).

There are several important points from these observations related to the modelling work. First, the X-ray luminosity seems to be varying roughly as $L_{x} \propto 1 / D$ where $D$ is the binary separation. This is in good agreement with the predictions of Stevens et al. (1992) for a widely separated binary such as HD 193793, where the post-shock gas is close to adiabatic. In this model the $\mathrm{X}$-ray emission is assumed to be thermal.

While this may be seen to be a strong confirmation of the basic colliding wind model, there is also some bad news for the standard model. The strength of the Fe- $\mathrm{K}$ line is very variable, and spectral fitting indicates the presence of powerlaw non-thermal emission in addition to thermal emission from the colliding wind shock. However, a non-thermal spectral component is not unexpected as the strong wind-wind collision shocks will be capable of accelerating electrons, resulting in non-thermal emission (Eichler \& Usov 1993). These observations of HD 193793 provide a view of a different range of high energy phenomena associated with colliding winds, and clearly represents a complication on the standard model requiring more investigation.

\section{Infrared line-profile variations in WR binaries}

To date I have emphasised the importance of X-ray data in understanding colliding winds, as X-rays uniquely sample the hot post-shock gas. However, work by Moffat, Marchenko, Lührs and others have shown that we can view the interaction region at optical wavelengths, and here I want to report on some phase resolved observations of the He I $10830 \AA$ line (Stevens \& Howarth 1999).

We observed the WR+O-star binary systems V444 Cyg, CQ Cep, CV Ser, GP Cep, and HD 193928 throughout their orbits from the INT on La Palma. Significant, systematic and similar line profile variations were observed in most systems. A montage of the data is shown in Figure 2. Concentrating on V444 Cyg (WR 139), we see that the emission line is basically flat-topped, but where peaks superimposed on it swing from blue to red-shifted and back through the orbit. Such variability is seen (to a lesser degree) in the other systems.

The He I $10830 \AA$ line emission could be coming from two sources in these binaries; first, the undisturbed wind of the WR star (which would tend to give a flat-topped profile) and second, the wind-wind interaction region. We do not expect any emission from the O-star wind, which will effectively comprise an "emission hole" moving round the orbit. However, a simple model, invoking just 


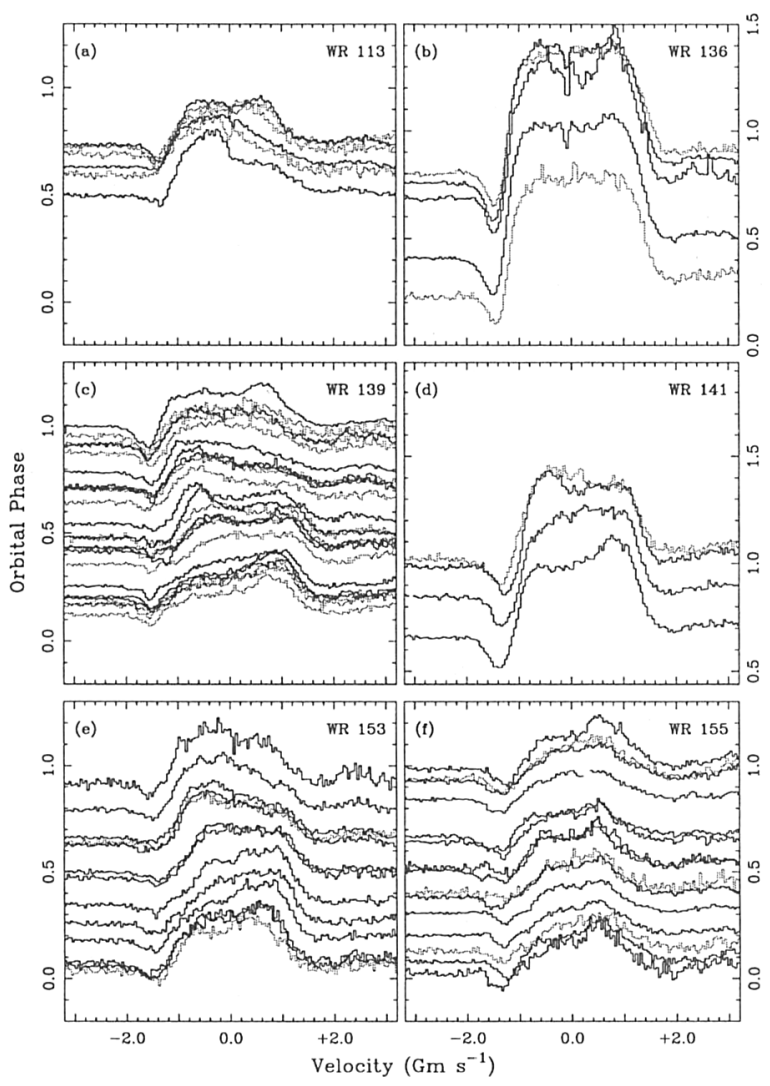

Figure 2. The orbital variability of the He 10830 line. Phase zero is defined as conjunction with the Wolf-Rayet star in front.

an absence of emission from the O-star wind cannot explain these observations, and so we conclude we are seeing emission from the wind-wind collision.

We have constructed a simple colliding wind model, based on the work of Lührs (1997) to explain these observations. In this model we have emission from the ambient wind of the WR star as well as emission from the wind-wind collision. In the original Lührs (1997) model the emission strength was uniform around the cone. In our model the two arms have unequal emission strength the trailing shock being stronger. This might be expected on physical grounds, the post-shock material being of a higher density. The original Lührs (1997) model predicts two moving bumps, whereas these observations suggest a single dominant bump. A slice in the orbital plane is shown in Figure 3, where we also show the expected phase variability for such a model, compared with the data for V444 Cyg. There is some general agreement between the model and observations in the broad variability, but there is also substantial disagreement in the details, which will take more refined models to understand.

These observations of the He I $10830 \AA$ line provide another handle on the wind-wind interaction, and pose certain questions. Do we only see the effects 

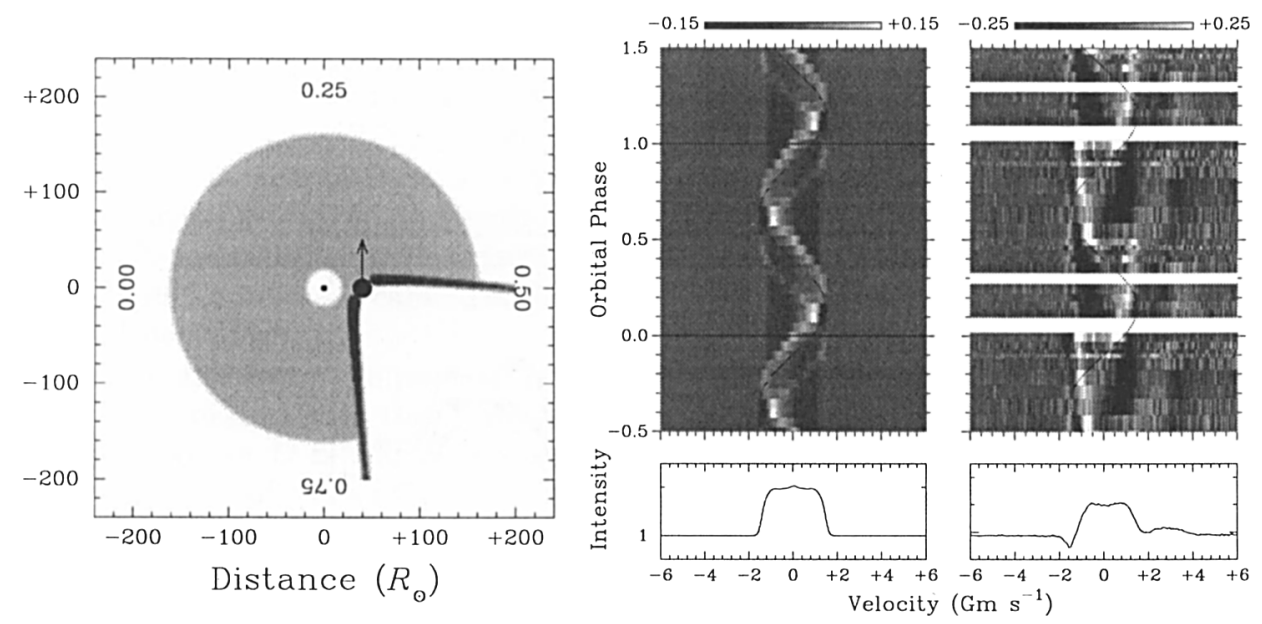

Figure 3. Left panel: An equatorial slice through a model including binary rotation and shock emission, showing the locations of distributed $\mathrm{He} \mathrm{I}$ emission from the WR wind and localized emission from the wind-wind interface. The arrow indicates the direction of binary motion of the O-star, and viewing directions for four binary phases are indicated. Middle panel: dynamic spectrum generated from difference spectra for the 'rotation+shock' model. Right panel: observations of WR 139 on a similar scale. A sine wave is drawn through both frames to emphasize the common 'S-wave' pattern in the emission.

of the wind-wind interactions in optical lines for close binary systems where the post-shock densities are sufficiently high to allow strong cooling? A systematic study could prove useful here.

\section{3D radiation hydrodynamic simulations}

All of the simulations used in the modelling work described in earlier sections have been axially symmetric $2 \mathrm{D}$-simulations. Some of them have included radiative driving, using a CAK type wind model (in the case of $\eta$ Car), while some of them have assumed terminal velocity winds (in the case of $\gamma^{2}$ Vel).

Under some circumstances these approximations are reasonable (wide binaries, such as HD 193793). But, for most systems of interest they are very poor approximations. In these systems it is clear that the inclusion of radiative driving is necessary and the inclusion of binary rotation is also necessary. Also, only by including radiative driving in such models can we understand the implications of effects such as sudden radiative braking on the X-ray emission (Gayley et al. 1997). We have been progressively developing more sophisticated colliding wind models, from the 2D terminal velocity simulations in Stevens et al. $(1992,1996)$, through the 2D-simulations including radiative driving (Pit- 


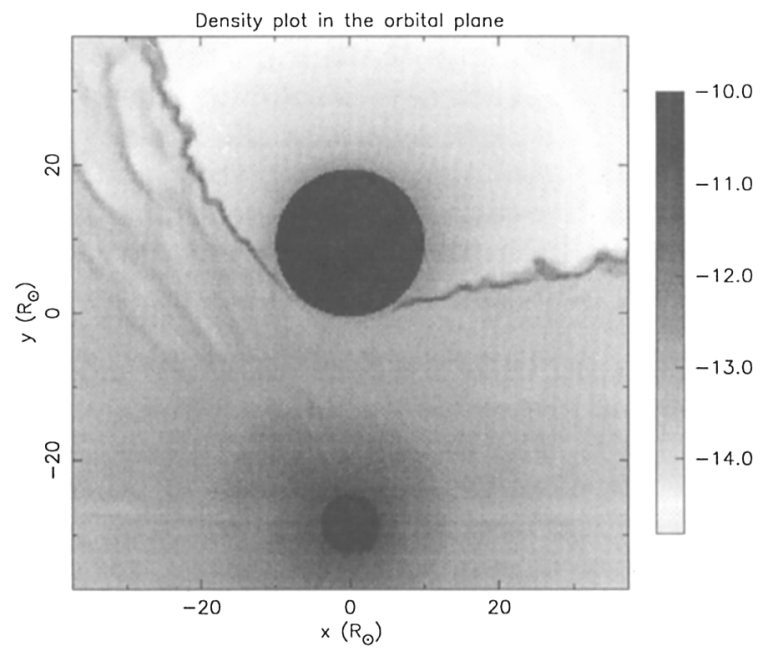

Figure 4. A density slice through the orbital plane of a 3D-simulation of V444 Cyg. The smaller Wolf-Rayet star has the stronger wind. The wind collision shock is visible, as is the aberration caused by the orbital motion.

tard 1998; Pittard et al. 1998) and now 3D-simulations with radiative driving (Pittard, these Proceedings). Some results from a simulation of V444 Cyg are shown in Figure 4.

These calculations are preliminary, but do include effects, such as radiative driving and binary rotation, and can be used as a basis for studies of the polarization in colliding wind systems or X-ray light curves.

\section{Future plans}

The next few years will be a particularly exciting time for the study of colliding winds, with the launch of $A X A F$ in 1999 and $X M M$ in 2000, which will open up new possibilities for studying colliding winds (a brief discussion of our plans for observing hot stars with XMM can be found in Rauw et al. - these Proceedings). Some possibilities include:

1. Spatially resolving colliding winds: The AXAF-HRC instrument has a spatial resolution of about $0^{\prime \prime} 5$. The colliding wind system WR 147 has two radio components (the WR star and the wind collision region) separated by 0.6 (Williams et al. 1997), and therefore potentially resolvable. AXAFHRC observations of WR 147 should be able to constrain the size and morphology of the colliding wind region, a possibility hitherto impossible. There is also the prospect of discovering other widely separated colliding wind binaries, also resolvable with AXAF ( $c f$. Wallace et al. - these Proceedings).

2. Spectrally resolving the velocity distribution of hot gas in the wind shock. The grating instruments on $A X A F$ and $X M M$ will have a velocity reso- 
lution of $\sim 1000 \mathrm{~km} \mathrm{~s}^{-1}$, comparable to the expected flow velocities in the post-shock gas. We should therefore see variations in the line-widths and line-centers of emission lines through the orbit, allowing us to study the dynamics of the post-shock flow in colliding winds.

3. The high spectral resolution of the grating instruments opens up the prospect of doing line-ratio studies to determine the details of the ther$\mathrm{mal} /$ density structure in the hot post-shock flow, as well as abundance analysis on many important elements.

4. The spectral imaging CCD detectors on $A X A F$ and $X M M$ will allow us to study the spectral properties of colliding winds over a large energy band (from $0.1-10 \mathrm{keV}$ ). This capability will be particularly important in that massive stars are often in close proximity to other stars, and there are situations where non-imaging detectors will have problems. The broadband spectral capabilities of both $A X A F$ and $X M M$ will allow the detection of any harder non-thermal components in both single and binary WR stars (cf. HD 193793, Skinner et al. 1997).

\section{Conclusion}

In this paper we have discussed some of the recent progress on the modelling of colliding winds, particularly in relation to the X-ray properties of colliding wind systems. The future prospects are very good with the imminent launches of $A X A F$ and $X M M$. The combination of sophisticated radiation hydrodynamic models with these observations will result in a much better understanding of winds in WR binaries.

\section{References}

Chapman, J.M., Leitherer, C., Koribalski, B., Bouter, R., Storey, M. 1999, ApJ in press Corcoran, M.F., Ishibashi, K., Davidson, K., Swank, J.H., Petre, R., Schmitt, J.H.M.M. 1997, Nature 390, 587

Corcoran, M.F., Petre, R., Swank, J.H., Drake, S.A., Koyama, K., Tsuboi, Y., Viotti, R., Damineli, A., Davidson, K., Ishibashi, K., White, S., Currie, D. 1998, ApJ 494,381

Damineli, A. 1996, ApJ 460, L49

Eichler, D., Usov, V. 1993, ApJ 402, 271

Gayley, K.G., Owocki, S.P., Cranmer, S.R. 1997, ApJ 475, 786

Lührs, S. 1997, PASP 109, 504

Pittard, J.M. 1998, MNRAS 300, 479

Pittard, J.M., Stevens, I.R., Corcoran, M.F., Ishibashi K. 1998, MNRAS 299, L5

Skinner, S.L., Itoh, M., Nagase, F. 1997, New Astr. 3, 37

Stevens, I.R., Blondin, J.M., Pollock, A.M.T. 1992, ApJ 386, 265

Stevens, I.R., Corcoran, M.F., Willis, A.J., Skinner, S.L., Pollock, A.M.T., Nagase, F., Koyama, K. 1996, MNRAS 283, 589

Stevens, I.R. Howarth, I.D. 1999, MNRAS 302, 549 
Williams, P.M., van der Hucht, K.A., Pollock, A.M.T., Florkowski, D.R., van der Woerd, H., Wamsteker, W.M. 1990, MNRAS 243, 662

Williams, P.M., Dougherty, S.M., Davis, R.J., van der Hucht, K.A., Bode, M.F., Setia Gunawan, D.Y.A. 1997, MNRAS 289, 10

Willis, A.J., Schild, H., Stevens, I.R. 1995, A\&A 298, 549

\section{Discussion}

Lepine: It is likely that stellar winds from $\mathrm{O}$ and WR stars are inhomogeneous; how do you think would a strong wind-clumping affect the physics and morphology of the collision region?

Stevens: Talking about X-rays, if the density contrast of the inhomogeneities is small then it won't be a major problem for the X-ray emission, as the shock will obliterate the blobs. Life becomes much harder if the density contrast is larger (which it could be). If the winds have substantial small scale structures, then it may be difficult to account for in our models.

Leitherer: There is no longer significant evidence for discrepancies between the radio mass-loss rate of WR 11 and those derived from other methods. The new value is $\dot{M}=2-3 \times 10^{-5} \mathrm{M}_{\odot} \mathrm{yr}^{-1}$. The downward revision is due to (i) the lower Hipparcos distance (van der Hucht et al. 1997, New Astronomy 2, 245) and (ii) some non-thermal contamination at $6 \mathrm{~cm}$. This value does not include a clumping factor.

Stevens: I'm very pleased to hear this.

Zhekov: For the radiative cases as WR 11 it is not correct to determine $\dot{M}$ from a comparison of models with observations, since the nowadays available HD models have problems with solving the singularity problem in the stagnation point. The problem is that these models use a cut-off cooling curve which means that below a given temperature there is no cooling. This is equivalent of introducing an oscillatory term in the energy equation and this changes the solution. That is, the X-ray spectrum is highly dependent on the cut-off temperature and the result is that the X-ray luminosity derived from the models might be very different and, therefore, incorrect.

Moffat: (1) If I remember correctly, the mass-loss rate of the WR star in $\gamma^{2}$ Velorum from polarization measurements (St-Louis et al. 1988) is $\sim 1 \times 10^{-5} \mathrm{M}_{\odot} \mathrm{yr}^{-1}$, a factor $2-3$ lower than both the radio and your X-ray values, which are affected by $\rho^{2}$ effects of clumping. The polarization observations are independent of both distance and clumping. (2) Maybe the reason why your He I $1.08 \mu$ wind collision line does not fit a simple model is due to the fact that this line has a strong $\mathrm{P}$-Cygni profile, and is complicated by other effects that I mentioned, that are less important in the C IV line of the non-eclipsing $\mathrm{WC}+\mathrm{O}$ systems which I discussed.

Yokogawa: The calorimeter onboard ASTRO-E is suitable for study of wind-wind collision (because it has a sensitivity up to $10 \mathrm{keV}$ and Fe-lines have been detected). Why do you like XMM and $A X A F$ better than ASTRO-E? AXAF and XMM will be launched earlier. ASTRO-E needs more time to accumulate enough photons than $A X A F$ and $X M M$ (a weak point for systematic study).

Stevens: The questioner has answered the question too. My answer is: ASTRO-E will be a very useful instrument too for colliding winds, if we can get time on it. I concentrate on $A X A F$ and $X M M$, because they will be launched sooner. 